\title{
Gender disparity within academic Canadian urology
}

Julius Ilin ${ }^{1}$; Emilie Langlois ${ }^{2}$; Sabeena Jalal ${ }^{3}$; Faisal Khosa ${ }^{3}$

${ }^{1}$ Faculty of Medicine, University of Ottawa, Ottawa, ON, Canada; ${ }^{2}$ Faculty of Medicine, University of Ottawa, Ottawa, ON, Canada; ${ }^{3}$ Department of Radiology, University of British Columbia, Vancouver, BC, Canada

Cite as: Can Urol Assoc J 2019 November 5; Epub ahead of print. http://dx.doi.org/10.5489/cuaj.6117

Published online November 5, 2019

$* * *$

\section{Abstract}

Introduction: Increasing female matriculation into medical school has shown an increase in women training in academic urology, but gender disparity still exists within this male-dominated field. This study aims to evaluate publication productivity and rank differences of Canadian female and male academic urologists.

Methods: The Canadian Residency Matching Service (CaRMS) was used to compile a list of 12 Canadian accredited urology programs. Using each institution's website, faculty members' names, genders, academic positions, and leadership ranks were noted. SCOPUS ${ }^{\odot}$ was consulted to tabulate the number of documents published, citations, and h-index of each faculty member. To account for temporal bias associated with the h-index, the m-quotient was also computed. Results: There was a significantly higher number of men $(164,88.17 \%)$ among academic faculty than women $(22,11.83 \%)$. As academic rank increased, the proportion of female urologists decreased. Overall, male urologists had higher academic ranks, h-index values, number of publications, and citations ( $\mathrm{p}=0.038, \mathrm{p}=0.0038, \mathrm{p}=0.0011$, and $\mathrm{p}=0.014$, respectively). There was an insignificant difference between men and women with respect to their $m$-quotient medians $(p=0.25)$.

Conclusions: There is an increasing number of women completing residency in urology, although there are disproportionally fewer female urologists at senior academic positions. Significant differences were found in the h-index, publication count, and citation number between male and female urologists. When using the m-quotient to adjust for temporal bias, no significant differences were found between the gender in terms of academic output. 


\section{Introduction}

The proportion of female students admitted to medical schools has significantly risen over the years. As such, entering medical school classes are at least $50 \%$ female. ${ }^{1}$ Along with this rise of female medical students, there has been a corresponding increase in women choosing the field of urology. However, in spite of the dramatic increase of women pursuing urology, they only represent $33 \%$ of students matching into this specialty. ${ }^{2}$ According to data gathered by The American Urologic Association, females represent $8.5 \%$ of total practicing urologists, though this number is expected to rise to $28 \%$ by $2050 .{ }^{3}$ Whilst there is movement towards equality in urology, there are still shortcomings in the gender equalization of staff positions and academic rank. Despite female urologists working more and publishing at a greater rate than male counterparts, there are fewer women obtaining senior leadership positions amongst academic physicians. ${ }^{4-6}$ A US study by Meyer et al. found that only $1.3 \%$ of female urologists occupy chair positions compared to $7.5 \%$ male. Similarly, $11 \%$ of female urologists compared to $26 \%$ of male urologists held professorship positions at academic centers. ${ }^{7,8}$ This paper investigates the gender differences in the relationship between academic productivity and career advancement for Canadian male and female urologists.

\section{Methods}

This was a retrospective cross-sectional study discerning gender and academic rank among university-affiliated urologists in Canada. Informed consent or institutional review board approval was not requested as all data are publicly available. The data collected was assembled from January 2017 to June 2019 through a method already validated through several recent publications. ${ }^{9-19}$ A database was generated by consulting the Canadian Residency Matching Service (CaRMS), through which a total of 13 accredited urology programs were eligible for investigation. Of the 13 aforementioned programs, 12 institutions had the required faculty listings on their website or provided a list of their faculty members through email. (Table 1).

Faculty members from the 12 programs were subsequently entered into a Microsoft Excel spreadsheet. Only physicians holding an M.D. and/or M.D./PhD degree with an appointment within the division of urology having completed a urology residency program in an accredited institution were included. Along with the faculty's name and gender, several other academic positions and leadership ranks were also noted. Gender identification was limited by name, faculty photo, or provincial college registration. SCOPUS (C) was consulted to gather information pertaining to the research productivity of each of the faculty members. Parameters recorded included number of documents published, citations, year of $1^{\text {st }}$ publication, year of most recent publication, years since $1^{\text {st }}$ publication, years since most recent publication, and $h$ index. The Hirsch $(h)$ index is a frequently used biometric that measures research productivity. Its computation uses both an author's quantity of publications as well as the number of citations. As such, it is both a qualitative and quantitative measure of an individual's research performance 
and has been correlated with academic rank. ${ }^{20}$ A limitation of this index is its inherent advantage for more senior researchers. Individuals conducting investigations for a longer duration would have had more opportunity to publish and thus more opportunity to be cited. To account for the temporal bias associated with the $h$ index, the $m$ quotient was computed for each faculty member. This biometric tool takes into consideration the duration of the researcher's publication career and is thus a more accurate depiction of a faculty member's research performance. ${ }^{21}$

\section{Results}

A total of 207 urologists, 179 men and 28 women, from 12 academic institutions across Canada were identified using faculty websites. Among these, academic rank and other variables were found and included for 186 staff members, 22 (11.83\%) women and 164 (88.17\%) men, which were included in the study (Table 2). For each of the academic ranks, the majority were males $82.05 \%$ of assistant professors, $91.84 \%$ of associate professors, and $93.22 \%$ of full professors. In fact, as the academic ranking increased, there was a decrease in the number of female faculty. Assistant professors included 14 women, and associate as well as full professor positions were held by 4 women each (Table 2). There was a significant difference in academic rank between the two genders $(p=0.038)$ as demonstrated by Table 3 .

The median publication count and range of publications was also assessed for the cohort of 186 urologists. For both males and females, the highest publication counts were observed in those with full professorship positions, wherein males had 140 (range $=13-578$ ) and females had a median of 69 (range $=14-279$ ) publications. The lowest median of publications was seen in the assistant professor category, in which men had a median of 22.5 (range =1-183) and women had a median of 9.5 (range $=2-54$ ) publications, as seen in Table 2 . There was a significant difference $(p=0.0011)$ between men and women for median and range of publications (Table 3 ). In the associate professor category, men had a median publication count of 59 (range $=2-287$ ) whereas women had a median publication count of 25.5 (range =16-55). As such, men had higher numbers of publications in all professorship categories.

Citations followed a similar pattern to publication production. Citations were highest among full professors, with men having a median of 3148 (range $=87-26671$ ) and women having 1267.5 (range $=384-10681$ ) citations. The lowest citation values were seen amongst assistant professors, with men having a median of 300.5 (range $=1-6267$ ) and women having a median of 68.5 (range $=3-545$ ) citations (Table 2 ). There was a significant difference $(p=0.014)$ between men and women with respect to citation median (Table 3$)$. Both publications and citations correlated with academic rank, a relationship that was observed for male and female urologists, although men had a higher median of citations in every professor rank (Table 2).

The $h$ index, incorporating both publications and citations, was used as a metric to assess the qualitative and quantitative research productivity of faculty members. The highest indices 
were seen among full professors, with men having a median of 31 (range $=5-91)$ and women 19 (range $=8-57$ ). The lowest indices were observed in the assistant professors, with men having a median of 8.5 (range $=1-45)$ and females 4.5 (range $=1-15)$, as seen in Table 2. There was a significant difference in the $h$ index between the two genders ( $p=0.0038$ ) (Table 3). As seen in Figure 1, both men and women follow a similar trajectory in terms of their $h$ indices - as academic rank increases, so does that $h$ index. Similarly, according to Figure 1, men had statistically higher $h$ indices in the assistant and associate professor rankings, but not in the full professor ranking due to the large error bars for women. Comparable to publications and citations, the associate professor rank saw males with a higher $h$ index than their female counterpart. In this category, men had a median of $17($ range $=1-45)$ compared to 10.5 (range $=$ 8-23) for women (Table 2).

The $m$ quotient is a reflection of the $h$ index while accounting for an individual's academic career length. Although the $h$ index is a universal marker of research productivity, it is associated with temporal bias as physicians with longer careers are more likely to have an increased number of publications and, consequently, citations. By including a physician's career duration as part of the computation of the index, the $m$ quotient normalizes the variability in career durations and standardizes research productivity. For both men and women, the $m$ quotient increased with academic rank (Figure 2). The large errors bars observed for women at full professor positions is due to the small sample $(n=4)$ of women in this category and the large variability of values. Men employed as full professors had an $m$ quotient median of 1.05 (range = 0.14-2.84) and women employed as full professors had an $m$ quotient median of 0.77 (0.32-1.90), as demonstrated by Table 2 . In the associate professor rank, men had an $m$ quotient median value of 0.86 (range $=0.051-2.05)$ and women had a median value of $0.72($ range $=0.35-0.96)$. Interestingly, in the assistant professor category with the largest number of women, men had a median $m$ quotient of 0.61 (range $=0.053-3.75$ ) and women had a slightly higher median $m$ quotient, that of $0.62(0.26-1.50)$ There was an insignificant difference between men and women with respect to their $m$ quotient medians $(p=0.25)$ as seen in Table 3 .

\section{Discussion}

Women have been historically underrepresented in academic medicine. This was due to the lack of female matriculation into medical school, a tendency that has been ameliorated over the years. Starting in 1969, the proportion of women enrolling in medical school began to significantly rise. ${ }^{21}$ This increase in matriculation saw a female medical school enrolment rate of $31 \%$ in 1981 augment to $47 \%$ in $2010 .{ }^{22}$ Surgical specialties began to see a similar increase in the proportion of females applying for residency positions. Amongst these, urology, a specialty dominated by men, has seen a substantially increasing pool of female applicants for residency positions, although women still remain a significant minority of the workforce. ${ }^{23}$ The proportion of female 
urologists in the United States has risen from $1.2 \%$ in 1995 to $8 \%$ in 2011, and is predicted to rise to $28 \%$ by 2050 . $^{7,24,25}$

The statistics in Canada follow a similar trend. Urology in Canada has become much more gender neutral over the years. Starting from the 1970s, that saw the graduation of the first woman from a Canadian urology residency, there has been an increase in the enrolment of women in medical schools. ${ }^{26,27}$ With the increase of the admittance of female students, CaRMS has seen a greater proportion of female applicants for urology as a first choice specialty. In 19981999 , the proportion of females applying to urology was $19 \%$ and increased to $27 \%$ in 2012 2015, which lead to $1 / 4$ of all Canadian urology residents becoming women. There was a similar trend in the proportion of female applicants to other surgical programs - 1998-1999 had a surgical cohort compromising of $25 \%$ women, whereas the proportion of women in surgical programs rose to $40 \%$ by $2012-2015 .{ }^{28}$

Career advancement is one metric that can be used to assess success in an academic centre. Our study, as indicated by Table 2, demonstrated that the absolute number of women decreases with advancing academic rank. Female urologists comprised $17.95 \%$ of assistant professors and only $8.16 \%$ and $6.78 \%$ of associate and full professors, respectively, and statistical analysis showed that academic rank differences between male and female urologists were significant $(p=0.038)$. These data are similar to those found in the United States, where men are more likely to occupy more senior positions and only $12 \%$ of female urologists see advancement past associate professorship, compared to $33 \%$ of male urologists. ${ }^{7}$ Academic output of the urologists was measured by the median number of publications and was found to have been significantly different between men and women $(p=0.0011)$, with male urologists having a median of 54.5 publications compared to 15 for female urologists, as shown by Table 2 and Table 3. A study conducted by Yang et al. in the United States found that women in urology have fewer publications than men in urology, although they are equally as likely to pursue fellowship training and choose an academic career. ${ }^{29}$ Given the results our study, it seems that Canadian female urologists follow a similar trend in reduced academic output.

To further evaluate research productivity, the $h$ index was computed for both genders in each cohort of professors. Upon computation of the $h$ index, there was a significant difference between men and women $(p=0.0038)$, similar to a finding in the United States where the median $h$ index of all male urologists was 11.67 , compared to 6.33 for female urologists. ${ }^{7}$ In order to reduce the temporal bias associated with the $h$ index, the $m$ quotient was computed. Since a longer career duration was anticipated for males thus resulting in a larger research output, the $m$ quotient's ability to negate temporal bias was crucial. Interestingly, the $m$ quotient demonstrated an insignificant difference $(p=0.25)$ between male (0.82) and female (0.72) urologists. This may indicate that the perceived superior academic output of male urologists, as demonstrated by their publication count and $h$ index, may simply be as a result of a temporal bias 
due longer careers. Because women have only started entering urology in larger quantities fairly recently, their academic output may be reduced. However, when adjusting for their relatively fewer years in practice, the $m$ quotient indicates that they are just as academically productive as their male counterparts.

There are several notions that may explain why females are often underrepresented in the upper echelons of academic medicine, most of which deal with a relative lack of research productivity, which can begin as early as residency training. Men have been shown to publish more during residency which thereby increases their research career duration, allowing them to begin developing their $h$ index sooner. ${ }^{29}$ Not only do women tend to publish less during residency, but they also may prioritize family responsibilities over academic goals. ${ }^{20}$ Taking time for maternity leave as well as child-rearing duties both substantially diminish the time attributed to academic pursuits, such as publications and career advancement. ${ }^{20,30}$

The precedent for contemplating a perceived gender gap in academic productivity may be alternatively related to recruitment, career length and practice settings. The lag effect of underrecruitment of women into urology is one major contributor to the issue. Before the increase of female matriculations in medical school, medicine and by extension urology, was a maledominated career. As female urologists began to enter the workforce, the faculties that recruited them had already had many other male urologists with long career durations. With longer career duration resulting in better measures of academic productivity, male urologists were more likely to see career advancement compared to the female urologists with relatively shorter career lengths. ${ }^{16}$ Women are also more likely to enrol in the clinician-educator rather than tenure track, and are hence more likely to prioritize teaching over academic research. This further reinforces lag effect in academic promotion for female urologists. ${ }^{25}$

Another issue faced by many female urologists is finding a mentor, a major setback compared to men. Mentors are crucial in exposing a student to a particular career field and in providing them with research opportunities. Many urology residents cite excellent mentorship as one of the top reasons to pursue urology. ${ }^{31}$ It has been noted that female medical students report facing difficulties in finding a mentor in surgical fields, whether of the same gender or not. This is often compounded by the male-dominant nature of urology, and thus disadvantages women interested in pursuing urology relative to their male counterparts, who are able to find a mentor more readily. Without a mentor or a mentor in leadership positions, women would not be exposed to senior-level leadership and would not be as prepared for senior positions as male urologists. $^{31-35}$ This may also explain why women in medicine often are less inclined to view leadership roles as desirable and even when these leadership roles are attained, women feel less self-efficacious than men. ${ }^{36}$

There are several limitations in this study. There was an inherent degree of potential inaccuracy in obtaining faculty listing from institutional websites as these may not be updated in 
real time. Moreover, the absence of a urologist on SCOPUSC was often associated with a lack of research production from said urologist. However, this may not necessarily be true. Moreover, name changes after divorce, marriage, or a change in institution can result in the unintentional incredibility of work. Furthermore, the faculty listing from L'Université de Montréal could not obtained. Although the institution was contacted in an attempt to extract faculty data, the university refused to provide their urology staff listing. Lastly, there is some innate bias in the $h$ index that highlights its limitations. The $h$ index does not offer any differentiation between the publication of numerous papers of poor quality versus a few papers of superior quality, and it does not discriminate between self-citations. ${ }^{37}$

\section{Conclusions}

In conclusion, this study demonstrates that gender disparity can be found within urology faculties across Canada. There is significant difference between men and women in academic rank ( $p=$ 0.038 ), with the proportion of women decreasing as academic rank increasing. This may be the result of a plethora of factors, namely men having longer research-oriented careers, the lag effect of under-recruitment of women in urology, and the difficulty female urologists have in finding a mentor. Similarly, the results demonstrate a significant difference in the median of publications and citations ( $p=0.0011$ and $p=0.014$, respectively), with men having considerably larger values in these categories. The $h$ index, a representation of research productivity was significantly higher in men $(p=0.0038)$, while the $m$ quotient, an indicator of research output accounting for temporal bias, did not show a significant difference between male and female urologists $(p=0.25)$. With the continued increase of women pursuing urology, understanding and analysing gender differences within the specialty is imperative. Namely, identifying and reducing the barriers that women face in achieving academic success is an instrumental consideration in order to achieve an optimally functioning work environment. 


\section{References}

1. Leslie K, Harriet WH, Patricia H, et al. "Women, minorities, and leadership in anesthesiology: take the pledge." (2017): 1394-96.

2. Spencer, ES, Allison MD, Nicholas RP, et al. "Gender differences in compensation, job satisfaction and other practice patterns in urology." The Journal of urology 195, no. 2 (2016): 450-55.

3. Kim SJ, Hyun G. MP15-04 Women in urology: time to lean in. J Urol. 2014;191:e145.

4. Reed DA, Enders F, Lindor R, et al. Gender differences in academic productivity and leadership appointments of physicians throughout academic careers. Acad Med. 2011;86:43-47.

5. Nonnemaker L. Women physicians in academic medicine- new insights from cohort studies. NEJM. 2000;342:399-405.

6. Weiss DA, Kovshilovskaya B, Breyer BN. Gender trends of urology manuscript authors in the United States: a 35-year progression. J Urol.2012;187:253-58.

7. Mayer EN, Sara ML, Heidi AH, et al. "Gender differences in publication productivity among academic urologists in the United States." Urology 103 (2017): 39-46.

8. Association of American Medical Colleges. Active Physicians by Sex and Specialty, 2015 (2015). https://www.aamc.org/data/workforce/reports/458712/1-3-chart.html. Accessed September 30, 2018.

9. Sheikh MH, Chaudhary AM, Khan AS, et al. "Influences for Gender Disparity in Academic Psychiatry in the United States." Cureus 10, no. 4 (2018).

10. Hamidizadeh, R, Jalal S, Pindiprolu B, et al. "Influences for Gender Disparity in the Radiology Societies in North America." American Journal of Roentgenology 211, no. 4 (2018): 831-38.

11. Shaikh AT, Farhan SA, Siddiqi R, et al. "Disparity in Leadership in Neurosurgical Societies: A Global Breakdown." World neurosurgery (2018).

12. Khan MS, Fatima K, Ayub MT, et al. "Women in Leadership Positions in Academic Cardiology: A Study of Program Directors and Division Chiefs." (2018): A103-A103.

13. Yang HY, Rhee G, Xuan L, et al. "Analysis of H-index in Assessing Gender Differences in Academic Rank and Leadership in Physical Medicine and Rehabilitation in the United States and Canada." American journal of physical medicine \& rehabilitation (2019).

14. Moghimi S, Khurshid K, Jalal S, et al. "Gender Differences in Leadership Positions Among Academic Nuclear Medicine Specialists in Canada and the United States." American Journal of Roentgenology 212, no. 1 (2019): 146-50.

15. Battaglia F, Shah S, Jalal S, et al. "Gender disparity in academic emergency radiology." Emergency radiology 26, no. 1 (2019): 21-28.

16. Shah A, Jalal S, and Khosa F. "Influences for gender disparity in dermatology in North America." International journal of dermatology 57, no. 2 (2018): 171-76. 
17. Battaglia F, Jalal S, and Khosa F. "Academic General Surgery: Influences for Gender Disparity in North America." Journal of the American College of Surgeons 227, no. 4 (2018): e126.

18. Ahmadi M, Khurshid K, Sanelli PC, et al. "Influences for gender disparity in academic neuroradiology." American Journal of Neuroradiology 39, no. 1 (2018): 18-23.

19. Khurshid K, Shah S, Ahmadi M, et al. "Gender differences in the publication rate among breast imaging radiologists in the United States and Canada." American Journal of Roentgenology 210, no. 1 (2018): 2-7.

20. Svider PF, Pashkova AA, Choudhry Z, et al. "Comparison of scholarly impact among surgical specialties: an examination of 2429 academic surgeons." The Laryngoscope 123, no. 4 (2013): 884-889.

21. Bornmann L, and Daniel HD. "The state of $h$ index research: Is the $h$ index the ideal way to measure research performance?." EMBO reports 10, no. 1 (2009): 2-6.

22. Association of American Medical Colleges. "US medical school applicants and students 1982-1983 to 2011-2012." (2012).

23. Halpern JA., Lee UJ, Wolff EM, et al. "Women in urology residency, 1978-2013: a critical look at gender representation in our specialty." Urology 92 (2016): 20-5.

24. Bradbury CL, King DK, and Middleton RG. "Female urologists: a growing population." The Journal of urology 157, no. 5 (1997): 1854-56.

25. Mayer AP, Blair JE, Ko MG, et al. "Gender distribution of US medical school faculty by academic track type." Academic Medicine 89, no. 2 (2014): 312-17.

26. Hill C. "On becoming the first woman urologist in Canada." Canadian Medical Association Journal 122, no. 3 (1980): 356.

27. Canadian Resident Matching Service. CaRMS: R-1 match reports. [updated 2015]. http://www.carms.ca/en/data-and-reports/r-1/. Accessed January 7, 2019.

28. Anderson K, Tennankore K, and Cox A. "Trends in the training of female urology residents in Canada." Canadian Urological Association Journal 12, no. 3 (2018): E105.

29. Yang G, Villalta JD, Weiss DA, et al. "Gender differences in academic productivity and academic career choice among urology residents." The Journal of urology 188, no. 4 (2012): 1286-1290.

30. Pagel PS, and Hudetz JA. "H-index is a sensitive indicator of academic activity in highly productive anaesthesiologists: results of a bibliometric analysis." Acta Anaesthesiologica Scandinavica 55, no. 9 (2011): 1085-1089.

31. Kerfoot BP, Nabha KS, Masser BA, et al. "What makes a medical student avoid or enter a career in urology? Results of an international survey." The Journal of urology 174, no. 5 (2005): 1953-57.

32. Kutikov A, Bonslaver J, Casey JT, et al. "The gatekeeper disparity—why do some medical schools send more medical students into urology?." The Journal of urology 185, no. 2 (2011): 647-52.

33. McGinty KL, Martin CA, DeMoss KL, et al. "Future career choices of women psychiatric residents." Academic Psychiatry 18, no. 2 (1994): 95-102. 
34. Bright CM. Duefield CA, and Stone VE. "Perceived barriers and biases in the medical education experience by gender and race." Journal of the National Medical Association 90, no. 11 (1998): 681.

35. Abu-Zaid A, and Altinawi B. "Perceived barriers to physician-scientist careers among female undergraduate medical students at the College of Medicine-Alfaisal University: A Saudi Arabian perspective." Medical teacher 36, no. sup1 (2014): S3-S7.

36. Pololi LH, Civian JT, Brennan RT, et al. "Experiencing the culture of academic medicine: gender matters, a national study." Journal of general internal medicine 28 , no. 2 (2013): 201-207.

37. Kreiner G. "The Slavery of the h-index-Measuring the Unmeasurable." Frontiers in human neuroscience 10 (2016): 556. 
Figures and Tables

Fig. 1.

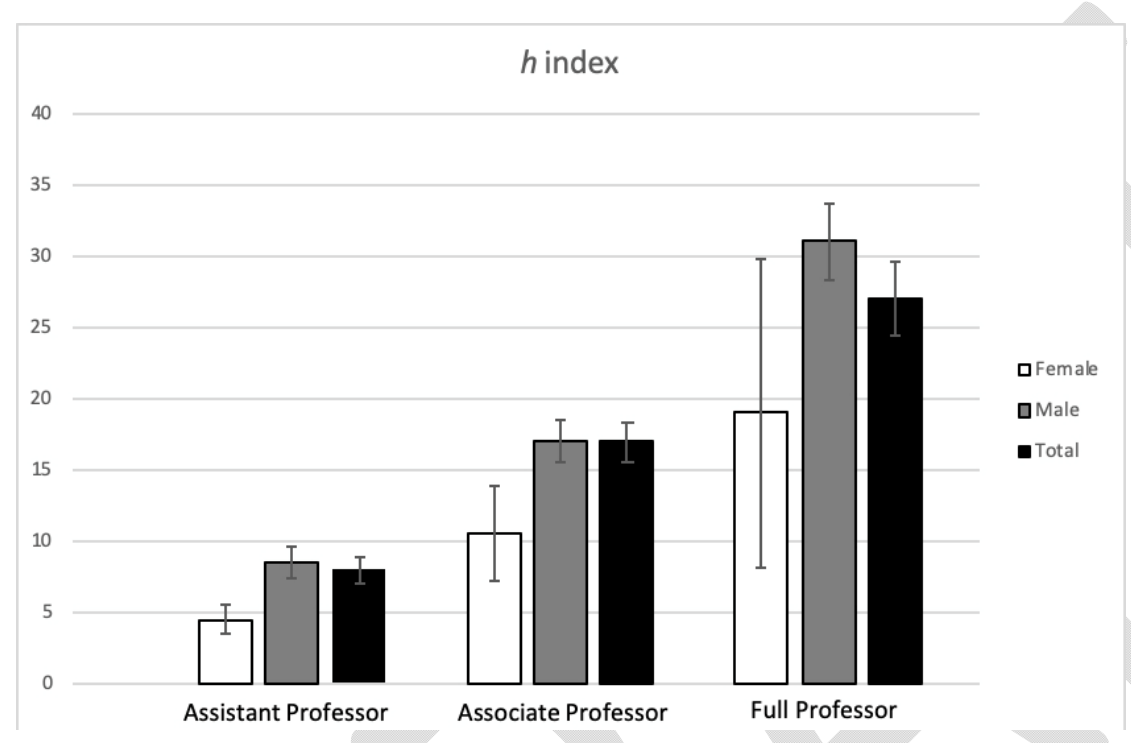

Fig. 2.

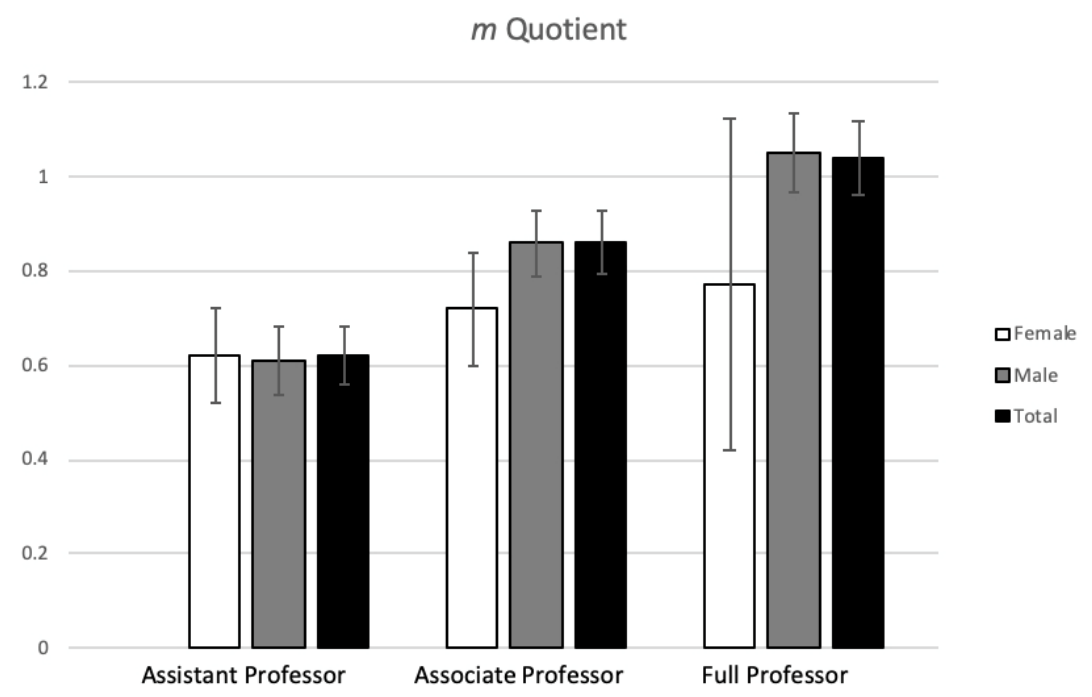




\begin{tabular}{|l|c|c|c|}
\hline Table 1. Gender breakdown amongst urologists across Canadian institutions \\
\hline Institution & Total no. faculty & Male no. faculty & $\begin{array}{c}\text { Female no. } \\
\text { faculty }\end{array}$ \\
\hline Dalhousie University & 12 & 9 & 3 \\
\hline University of Ottawa & 15 & 15 & 0 \\
\hline Queen's University & 10 & 10 & 0 \\
\hline University of Toronto & 28 & 27 & 1 \\
\hline McMaster University & 14 & 14 & 0 \\
\hline Western University & 12 & 12 & 0 \\
\hline University of Manitoba & 8 & 6 & 2 \\
\hline University of Alberta & 8 & 7 & 1 \\
\hline University of British Columbia & 27 & 24 & 3 \\
\hline McGill University & 18 & 17 & 1 \\
\hline L'Université de Sherbrooke & 6 & 5 & 10 \\
\hline L'Université Laval & 28 & 18 & 22 \\
\hline Total & 186 & 164 & \\
\hline
\end{tabular}

Table 2. Academic research activity and career duration for urology faculty by gender across Canada.

\begin{tabular}{|l|c|c|c|c|c|}
\hline $\begin{array}{l}\text { Position \& } \\
\text { gender }\end{array}$ & No. (\%) & $\begin{array}{c}\text { Publications, } \\
\text { median } \\
\text { (range) }\end{array}$ & $\begin{array}{c}\text { h-index, } \\
\text { median } \\
\text { (range) }\end{array}$ & $\begin{array}{c}\text { Citations, median } \\
\text { (range) }\end{array}$ & $\begin{array}{c}\text { m-quotient, } \\
\text { median (range) }\end{array}$ \\
\hline $\begin{array}{l}\text { Full professor } \\
\text { Male }\end{array}$ & $55(93.22 \%)$ & $140(13-578)$ & $31(5-91)$ & $3148(87-26671)$ & $1.05(0.14-2.84)$ \\
Female & $4(6.78 \%)$ & $69(14-279)$ & $19(8-57)$ & $1267.5(384-10681)$ & $0.77(0.32-1.90)$ \\
\hline $\begin{array}{l}\text { Associate } \\
\text { professor }\end{array}$ & 49 & & & & \\
Male & $45(91.84 \%)$ & $59(2-287)$ & $17(1-45)$ & $890(3-6355)$ & $0.86(0.051-2.05)$ \\
Female & $4(8.16 \%)$ & $25.5(16-55)$ & $10.5(8-23)$ & $450(233-656)$ & $0.72(0.35-0.96)$ \\
\hline Assistant professor & 78 & & & & \\
Male & $64(82.05 \%)$ & $22.5(1-183)$ & $8.5(1-45)$ & $300.5(1-6267)$ & $0.61(0.053-3.75)$ \\
Female & $14(17.95 \%)$ & $9.5(2-54)$ & $4.5(1-15)$ & $68.5(3545)$ & $0.62(0.26-150)$ \\
\hline Total & 186 & & & & \\
Male & $164(88.17 \%)$ & $54.5(1-578)$ & $16(1-91)$ & $796(1-26671)$ & $0.82(0.05-13.75)$ \\
Female & $22(11.83 \%)$ & $15(2-279)$ & $8(1-57)$ & $188.5(3-10681)$ & $0.72(0.26-1.90)$ \\
\hline
\end{tabular}


Table 3. $p$ values for gender differences in academic productivity measures for urology faculty across Canada

\begin{tabular}{|l|c|c|c|c|c|}
\hline & Academic rank & Publications & Citations & h-index & m-quotient \\
\hline $\mathrm{p}$ & 0.038 & 0.0011 & 0.014 & 0.0038 & 0.25 \\
\hline
\end{tabular}

